MEI

II, vol. 3

$n^{0} 4$

\section{Coaching documental: un nuevo paradigma para la profesión}

\author{
Empar Cuellar Alejandro* y A. Mario Gato Gutiérrez* \\ *Universitat Politècnica de Valencia
}

\title{
Resumen
}

Esta ponencia resume y presenta un nuevo reto para la profesión del documentalista. Este reto consiste en hacerse un nombre dentro la gestión de la documentación en las pequeñas y medianas empresas, hablando su mismo lenguaje. Este tipo de organización no se puede permitir tener un documentalista o gestor de información a tiempo completo por eso se debe formar al personal para que puedan cubrir sus necesidades informativas y organizacionales. Se pretende definir las herramientas y habilidades que debe tener toda persona que se quiera dedicar a esta rama profesional. Es una traslación de la formación de usuarios dentro del ámbito de las bibliotecas, aplicada al mundo de la empresa.

\section{Palabras clave}

Formación, Salida Profesional, Empresa, Usuarios.

Documentary Coaching : a new paradigm for the profession

\section{Abstract}

We try to explain a new challenge for the profession of librarian. The challenge is give a name to be understood by small and medium enterprises, it is speaking their language. This type of organization can not afford a librarian or an information manager full time so you should train staff so they can meet their needs and organizational information. We define the tools and skills that are accorded to people who want to dedicate this occupational field. Is a translation of user training in the field of libraries, applied to the business world.

\section{Keywords}

Training, Professional opportunities, Enterprise, Users.

\section{Introducción}

Varias son las labores de las nuevas generaciones que nos vamos incorporando a este apasionante mundo de la bibliotecología y la documentación como la de ser un relevo generacional digno, la de aportar nuevas formas de actuación y contribuir a la solución de nuevos retos, todo ello para que esta profesión cumpla las expectativas que la sociedad siempre ha puesto en ella.

A continuación, vamos a presentar una evolución natural de nuestra profesión, una nueva forma de adaptarse a los cambios tecnológicos, así como a la nueva cultura empresarial. Con este artículo se pretende dotar de un nuevo lenguaje a la profesión del gestor de información, para que la difusión de nuestras competencias sea óptima. Con todo esto se quiere dar una nueva salida profesional a los nuevos titulados, así como adaptar 
nuestra formación académica a los requisitos que quiere la sociedad en la que estamos inmersos.

Desde siempre los profesionales de la documentación hemos sido los encargados de entrenar y asesorar a la sociedad en su búsqueda de conocimiento. El coaching es, básicamente, lo mismo, entrenar a una persona para que consiga una serie de metas. Esto es lo que siempre se ha hecho en los programas de formación de usuarios. A lo largo del artículo se verá que nuestra profesión ya tiene integrados muchos de los conceptos y habilidades que ha de tener un coach o entrenador. Otra analogía es la del entrenador de un deportista, siempre está a su lado asesorándolo para que llegue al máximo de su potencial mediante su esfuerzo personal.

\section{Realidad social}

Nuestra labor desde siempre se ha desarrollado en unos ABCD muy definidos, el clásico Archivos, Bibliotecas y Centros de Documentación. Está clasificación siempre ha encorsetado a los profesionales en unas determinadas áreas. Siempre se han tenido unos roles bien definidos como son el de bibliotecario, el de servicio de referencia, el de formación de usuarios. Esto es una carga muy fuerte que arrastra nuestra profesión ya que el mundo empresarial siempre ha encasillado en áreas muy concretas a nuestros profesionales.

Para poder vender productos en el mundo empresarial hay que hablar su mismo lenguaje. Por eso debemos cambiar una serie de términos que como profesionales sólo debiéramos usar dentro del ámbito académico. Uno de esos términos es el de "formación de usuarios". Muchas empresas de servicios sí que tienen claro quién es el usuario, pero para otro tipo de empresarios que no tienen como clientes a personas físicas, el concepto de usuario les resulta extraño, suelen entender mejor el símil de cliente y proveedor.

También al usar la misma terminología que las empresas conseguiremos una mayor visibilidad de nuestra labor, de lo que podemos ofrecer y por tanto tener más posibilidades de ser contratados.

En nuestro país la mayor parte del tejido industrial y productivo está constituido por pequeña y mediana empresa. Una de las peculiaridades de este tipo de organizaciones es que suelen tener menos de 500 trabajadores y con unas cadenas de mando muy pequeñas. También el propietario tiende a ser el responsable de comunicación, delegando en algún administrativo la gestión de la documentación.

Una de las cosas que no se suele delegar es la política de comunicación de la empresa. Piensan antes en psicólogos, periodistas e informáticos para que lleven esa área aunque lo más habitual es que sea el propio empresario el que lleve a cabo esas tareas, negando cualquier posibilidad a la externalización del servicio, es decir, negando una salida profesional.

Aquí es donde entra el coach documental. La persona encargada de formar y 
asesorar a la organización tanto en sus tareas de gestión documental como en el desarrollo de sus políticas respecto al mundo 2.0 que nos envuelve. Así, el empresario sigue desarrollando la política de comunicación de su empresa sin necesidad de externalizar el servicio.

Esto es lo mismo que llevar la formación de usuarios y la alfabetización informacional al mundo de la empresa pero con un nombre que lo hace atractivo. Esto es básico pues, como hemos dicho antes, existe un desfase entre nuestra terminología y la empleada en el mundo de la empresa.

Otro de los problemas que tiene nuestra profesión es el intrusismo. Debido a la baja visibilidad que tenemos fuera del mundo de la Administración Pública, muchos colectivos afines como son informáticos, psicólogos, periodistas y diseñadores gráficos se han atribuido funciones que son propias de nuestra profesión.

\section{¿Qué es el coaching?}

Visto todo esto vamos a definir lo que se entiende por coaching. El concepto proviene del verbo inglés coach (entrenar) y se entrena para resolver las situaciones a las que nos enfrentamos. Por eso, el coach no enseña, sino que asiste al coachee (entrenado) a lograr un aprendizaje por sí mismo. Esto ya nos recuerda a uno de los principios que ha de tener un buen servicio de referencia: asesorar sobre la información que se tiene que buscar.

Otra definición más académica sería la de un proceso interactivo a través del cual un coach (entrenador) asiste al coachee (cliente que recibe el coaching) a obtener lo mejor de sí mismo. El coach ayuda a la persona a alcanzar ciertos objetivos fijados, utilizando sus propios recursos y habilidades de la forma más eficaz. Es una especie de consultor o mentor que permanece siempre disponible, que nos ayuda a modificar comportamientos y aptitudes; competencias y conductas.

Aunque hablamos de un concepto relativamente nuevo, se puede relacionar el coaching con la manera de pensar y actuar de filósofos de la Antigua Grecia como son Sócrates y Platón. Sin embargo, es a principios de los años noventa cuando se funda la primera escuela de formación de coaches, en América. Esta disciplina es nueva y en España no cuenta con estudios reglados, todo se deja en manos de empresas que acreditan mediante títulos propios la tenencia de dichas habilidades profesionales.

Pero el coaching no sólo está orientado al mundo empresarial, porque, según las distintas escuelas existentes, permite el crecimiento tanto profesional como personal.

La aplicación se consigue a través de un sistema de preguntas, que se realizan desde la aceptación y no desde el juicio, y que descubre cuáles son los objetivos y cómo lograrlos. Mejora la imagen que tenemos de nosotros mismos y profundiza de manera positiva en las relaciones interpersonales.

El coaching puede definirse como "un espacio privado" entre el interlocutor y su 
coach. Sin interferir en su entorno, el coach consigue que el interlocutor identifique los aspectos problemáticos de su gestión profesional y laboral. Le ayuda a fortalecer su confianza, aumentando su poder de liderazgo y su capacidad comunicativa. Y lo que no es menos importante, consigue que el interlocutor realice un análisis personal que le permita tomar las mejores decisiones. Se trata de una alianza entre el coach y su cliente. Un acuerdo entre dos partes en el que se establecen los objetivos que se necesitan alcanzar. Requiere establecer un programa y una agenda de trabajo con fechas, horarios, etc. Y una evaluación de resultados que el cliente va consiguiendo durante el proceso de coaching. La comunicación debe ser clara y de absoluta confianza. Se trata de un proceso de feedback sobre las conductas observadas.

Una definición más exacta es la que presentan Menéndez y Worth (2002) autores del libro 'Abre el melón', definen el coaching como "una serie de técnicas y procesos que te ayudan a realizar mejor todo aquello que ya sabes hacer, potenciando todas las habilidades y capacidades y, al mismo tiempo, permite el aprendizaje de conceptos necesarios para llegar hasta donde desees." Este libro es el que dio inicio a toda la filosofía coach.

El coaching requiere de cinco pasos básicos: la observación (nuevos puntos de vista, análisis de paradigmas, creencias y conductas), la toma de conciencia (reflexión sobre el poder de la elección y sus consecuencias), la determinación de los objetivos (definir qué es lo quiere lograrse), la actuación (con la información ya reunida, el coachee debe actuar de una forma sostenida en el tiempo) y la medición (es necesario comprobar si el objetivo perseguido está cerca de las expectativas del coachee).

Menéndez y Worth (2002), en su libro 'Abre el melón' definen 7 fases, algunas coincidentes con las de Mario Díaz: rapport (armonía entre coach y coachee), exposición, definir objetivos, observación, feedback, compromiso, y la toma de decisiones. Son en estos últimos 7 puntos en los que nos vamos a basar para establecer las pautas de actuación que debe tener un buen coach documental.

\section{Definición de las reglas del coaching documental}

El coaching es una forma interactiva de ayudar a los demás (personas, empresas, organizaciones), a desarrollarse rápidamente con la finalidad de conseguir resultados óptimos, tanto en el campo 2.0 como en el de la gestión documental. Hay que tener en cuenta que se puede aplicar a todo el personal de la organización (todo el mundo debe tener en cuenta que cada persona de la organización debe saber el verdadero valor de una buena gestión documental, también el valor de una buena política de comunicación).

Es un profesional de la "escucha activa” y de la observación. Estas son también cualidades que se le suponen a buen profesional de un servicio de referencia. Entre el coach y el coachee (cliente) se crea un ambiente de confidencialidad. Un coach profesional ayuda al coachee a: 
- Definir objetivos.

- Elaborar estrategias.

- Utilizar sus recursos.

- Tomar decisiones.

- Enfrentarse a situaciones difíciles.

- Desarrollar las actividades profesionales.

El contacto con el coach puede ser directo y a través de la teleformación, en la cual se emplean el teléfono e Internet entre otros materiales pedagógicos. Afortunadamente el dominio de las nuevas tecnologías no es algo ajeno a nuestra profesión. El coach es la persona donde se reflejan los contrasentidos del cliente, lo que le ofrece a éste una forma diferente de comprender cómo se alinean su propia personalidad, sus roles, sus actos y sus resultados.

Las cualidades que debe tener una persona de nuestra formación que quiera desarrollar su carrera profesional como coach documental se resumen en los siguientes puntos:

- Debe tener empatía y ser capaz de establecer un clima de tranquilidad con su cliente.

- Tener buenas dotes de comunicación, tanto para expresar las ideas como para estructurar la transmisión de conocimiento de forma óptima, procurando que las emociones no influyan en ese proceso.

- Debe tener una mente abierta y capaz de preguntar y responder con rapidez, obteniendo así mucha información valiosa.

Tabla 1. Habilidades del $\operatorname{coach}$ (fuente: Menéndez y Worth, 2002)

\begin{tabular}{|l|l|}
\hline \multicolumn{2}{|c|}{ HABILIDADES DEL COACH } \\
\hline - Armonía / rapport (relación) & - Metáforas. \\
- Articular lo que está pasando. & - Mantener la agenda del cliente. \\
- Automanejo de las emociones, del tiempo. & - Mantenerse enfocado en el coaching. \\
- Brainstorming (tormenta de ideas, creatividad). & Paciencia. \\
- Claridad. & - Pedir permiso. \\
- Confidencialidad. & - Preguntas poderosas (preguntas abiertas: \\
- Crear confianza. & cómo, qué, cuándo). \\
- Desafío. & - Reconocer los resultados, ser consciente de \\
- Escuchar. & ellos. \\
- Establecer y planear metas. & - Reencuadre. \\
- Estructura. & - Responsabilidad (dar importancia a los lo- \\
- Fondo de la cuestión. & gros obtenidos). \\
- Imparcialidad. & - Silencio. \\
- Interés. & - Solicitar información. \\
- Interrupciones con tacto, demostrando qué es & - Uso del lenguaje del cliente. \\
importante. & - Validación de los valores del cliente. \\
- Intuición. & - Ver desde una posición de meta. \\
- Llevar a cabo la acción. & - Visión del objetivo a alcanzar. \\
& - Visualizar los problemas. \\
\hline
\end{tabular}


Como se puede observar un buen profesional de la gestión documental debe tener muchas de las cualidades que están ahí expuestas. Sólo hay que pensar en las cualidades que debe reunir el personal que se encuentra en un servicio de referencia.

A diferencia de un consultor o especialista, el coach no necesita tener conocimientos específicos de temas referentes a la consultoría o la psicología. Tampoco debe tener experiencia en el tema del que tratará con su cliente. Sí debe estar convencido de que lo que ofrece es el mejor camino, de que su cliente será capaz de conseguir sus objetivos.

Todo lo dicho anteriormente se resume en la siguiente metodología: el coaching utiliza las entrevistas para profundizar en la comunicación interpersonal, los estilos de comportamiento, la capacidad de liderazgo y la toma de decisiones de su cliente. Todo ello mediante un diálogo privado basado en la confianza y la confidencialidad. Las entrevistas se llevan a cabo mediante e-mail, conversaciones telefónicas, chat o presenciales. La frecuencia, los horarios y el lugar de dichas entrevistas se acuerdan entre coach e interlocutor.

Es importante que el interlocutor esté dispuesto a ver las cosas con nuevas expectativas. A reconocer cuáles son sus errores, de modo que el coach pueda proporcionarle aquello que sea necesario para mejorar su situación.

\section{Etapas del proceso de coaching}

1.- Explicar el propósito y la importancia de lo que se está tratando de enseñar.

2.- Explicar los procesos.

3.- Mostrar a las personas los modos de realización y las técnicas que deben utilizar.

4.- Observar mientras las personas practican el proceso.

5.- Proveer retroalimentación inmediata y específica, ya sea para corregir errores o para reforzar éxitos.

6.- Expresar confianza en la habilidad de la persona para que tenga éxito.

7.- Llegar a acuerdos con las personas en relación con acciones de seguimiento.

\section{Proceso de coaching personalizado en siete etapas}

A continuación vamos a desarrollar la metodología que debe llevar a cabo el coach ya sea para asesorar documentalmente a la empresa o para intentar que se lleven a cabo políticas de comunicación en el mundo 2.0.

\section{1.- Rapport, armonización o empatía:}

Es importante tener armonía, eso es el rapport. Si el cliente no crea un buen ambiente con su coach, se aconseja que busque otro hasta encontrar aquel o aquella con quien se sienta más a gusto.

\section{2.- Exposición:}

A diferencia de la terapia y/o la consultoría, en el coaching no es necesario profundizar en las raíces de un problema. El bloqueo, a veces, lo produce el hecho de querer 
saberlo todo. Simplemente, se habla de aquello que se quiere tratar, y del por qué se necesita un coach. Para ello, sí que es necesario tener voluntad para abrir la mente y andar el camino (lo que nos recuerda un verso de A. Machado: "se hace camino al andar").

\section{3.- Definir objetivos:}

El cliente debe marcar el camino por el que "andará" para conseguir sus objetivos. Y debe hacerlo en positivo, mencionando tan sólo aquello que quiere alcanzar y no lo que no quiere. Debe ser específico. Una vez haya establecido la meta, debe ofrecer el máximo de detalles de los componentes que se incluyen en su objetivo. El objetivo debe ser realista, concreto y cuantificable. Hay que definir los objetivos para hacer una programación por etapas. De este modo, según avancemos por el camino marcado, iremos comprobando la etapa por la que nos vamos encontrando. El coach ayuda a su cliente a pensar, a establecer enlaces en el proceso de su vida. Actuando como un catalizador de sus pensamientos.

\section{4.- Observación:}

El coach ayudará a su cliente a considerar opciones que pudieran habérsele ocurrido antes, pero que no se atrevió a intentar. El cliente irá observando todo aquello que ocurra o sienta según vaya avanzando.

\section{5.- Feedback:}

El coach ofrece a su cliente un feedback objetivo y positivo, aún cuando su cliente sea subjetivo en su propio feedback, además de negativo. Este punto es muy importante, porque es una forma de ver si se está entendiendo el mensaje y de efectuar comprobaciones durante el proceso.

\section{6.- Compromiso:}

De nada le servirá al cliente todo lo anteriormente expuesto si no se compromete a realizar aquello que se establece durante el coaching. El cliente debe adquirir el compromiso de buscar y encontrar aquello que le frena durante su camino hacia la meta establecida, de este modo, logrará superar las barreras para seguir avanzando.

\section{7.- ¡Toma decisiones!}

Tal y como hemos dicho antes, la toma decisiones es una competencia exclusiva del cliente. Aquí podemos ofrecer un servicio de asesoramiento, como expertos que somos en materia documental y el mundo 2.0, de las iniciativas emprendidas. De nada sirve el entrenamiento que hacemos con nuestro cliente, si él mismo no se atreve a tomar las acciones para cambiar su situación actual.

\section{Conclusiones}

Por último queda definir el coach documental como ese profesional que se va a dedicar a asesorar empresas, particulares y trabajadores sobre los distintos retos que quieran adquirir en el mundo 2.0 o en el ámbito de la gestión documental, siguiendo 
las normas que hemos presentado anteriormente.

A modo de resumen el buen coach documental será aquella persona que consiga que sus coachees alcancen sus objetivos profesionales, mediante una planificación seria y un entrenamiento riguroso. Es decir, aplicamos el símil de enseñar a pescar, en vez de dar el pescado a nuestros clientes para que consigan saciar su hambre.

Esta nueva forma de ver nuestra profesión con respecto al mundo empresarial y las nuevas tecnologías tiene que ser una meta para que los nuevos profesionales consigan nuevos nichos de mercado donde desarrollar una carrera profesional, ya que los sitios clásicos están saturados.

Como se puede observar muchas de las competencias que se le piden a un coach van implícitas en la formación que recibimos en las universidades. Hay que abrir nuevas vías para el desarrollo de nuestra profesión y la conquista de nuevas áreas en las que ser útiles a la sociedad. Además si se consigue una mayor visibilidad, se combatirá mejor el intrusismo y se consolidarán los campos profesionales en los que no están bien definidas nuestras competencias.

La existencia de formación reglada, con titulaciones oficiales, se puede convertir en un punto a favor para poder incorporar a nuestro currículo profesional dichas habilidades.

Esperamos que con lo dicho anteriormente se haya abierto una nueva forma de ver las distintas facetas de nuestra profesión aplicadas al mundo empresarial y que todo esto contribuya a una mayor visibilidad y comprensión por la sociedad de todos los beneficios que nuestra titulación aporta.

\section{Referencias bibliográficas}

Anzorena, O. (2008), Maestría Personal, El camino del Liderazgo - Un modelo para la práctica del coaching y la facilitación del Desarrollo Personal y Organizacional, Lea, Buenos Aires.

Escribano, C. (1999), Psicología para un nuevo estilo de mando, Pirenaica, Zaragoza.

Flores, F. (1994), Creando Organizaciones para el Futuro, Dolmen, Madrid.

Menéndez, J.L., Worth, Ch. (2002), Abre el melón: cómo te puede ayudar el coaching o conseguir tus metas, Aguilar, Madrid.

Ortiz de Zárate, M. (2010), "Psicología y Coaching: marco general, las diferentes escuelas”, Capital Humano, No 243. 